\title{
Comparison of Attachment Styles, Resilliency and Social Capital in the Spouses of Martyrs, Veterans and Normal People; A Case Study of Ardabill City
}

\section{ART ICLE INF O}

\section{Article Type}

Descriptive Study

\section{Authors}

Abbasi Abrazgah M.* MSC, Khodaee SH. ${ }^{1} M S c$

How to cite this article
Abbasi Abrazgah M, Khodaee SH.
Comparison of Attachment Styl-
es, Resiliency and Social Capital
in the Spouses of Martyrs, Vete-
rans and Normal People; A Case
Study of Ardabil City. Iranian Jo-
urnal of War \& Public Health. 2018
$; 10(4): 209-214$.

*Psychology Department, Humanities Faculty, Malayer University, Malayer, Iran

${ }^{1}$ Psychology Department, Humanities Faculty, Ardabil Branch, Islamic Azad University, Ardabil, Iran

\section{*Correspondence}

Address: Malayer University, Kilometer 4 Malayer Road, Arak, Iran. Postal Code: 6571995863

Phone: -

Fax: -

mozhganabbasiabrazgah@gmail.com

\section{Article History}

Received: April 16, 2018

Accepted: August 04, 2018

ePublished: October 11, 2018

\section{A B S T R A C T}

Aims War is a complex social event that affects institutions and social life. The purpose of this study was to compare the attachment styles, resilience and social capital in the spouses of witness, veterans and normal people.

Instruments \& Methods This descriptive cross-sectional study was conducted in 2016 among the spouses of witness, veterans and normal people in Ardabil city. Using cluster sampling, 135 people (45 people in each group) were selected as sample. For data collection, Avdnhavn Attachment Questionnaire, Connor-Davidson Resilience Scale and Social Capital Questionnaire based on the Nahapit and Ghoshal model were used. The data were analyzed by Manova test and LSD test, using SPSS 16 software.

Findings There was no significant difference among three groups in secure attachment $(\mathrm{F}=0.123 ; \mathrm{p}=0.884)$ and avoidance attachment $(\mathrm{F}=0.122 ; \mathrm{p}=2.136)$, but there was a significant difference among fearful attachment scores ( $\mathrm{F}=3.164 ; \mathrm{p}=0.045)$. Also, there was a significant difference among three groups in resilience scores $(F=3.770 ; p=0.026)$ and social capital scores $(\mathrm{F}=4.715 ; \mathrm{p}=0.011)$.

Conclusion The fearful attachment style of veterans' spouses is more common than normal spouses, but other attachment styles are the same in all three groups. Also, the level of resilience and social capital are different in three groups; So that the resilience of spouses of normal people is higher than veterans' spouses. The spouses of witness and veterans comparing to normal spouses have higher social capital.

\section{Keywords Resilience; Social Capital; Spouses; Veterans}

\section{I T A T I O N L I N K S}

\begin{tabular}{|c|}
\hline 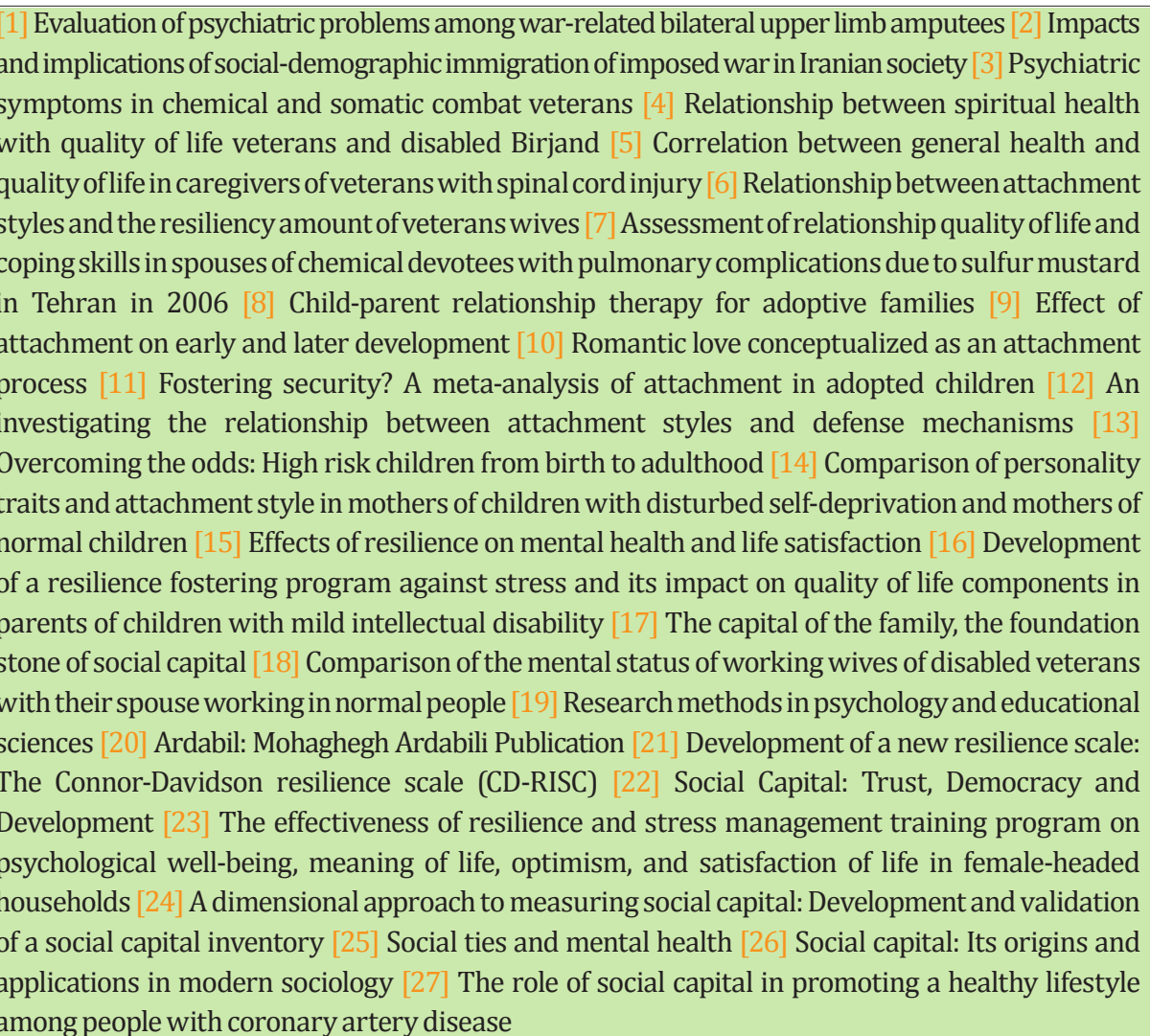 \\
\hline
\end{tabular}

Copyright(C) 2018, ASP Ins. This open-access article is published under the terms of the Creative Commons Attribution-NonCommercial 4.0 International License which permits Share (copy and redistribute the material in any medium or format) and Adapt (remix, transform, and build upon the material) under the Attribution-NonCommercial terms. 
روريرست خانواده يا حضور ناكارآمد او بهدليل معلوليت جسمى يا

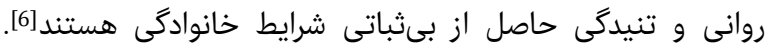

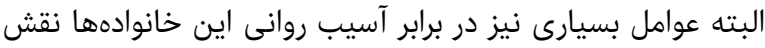

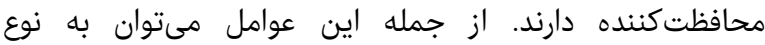

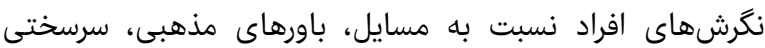

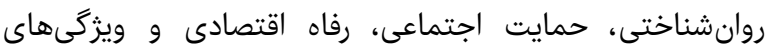

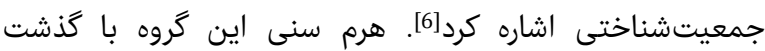

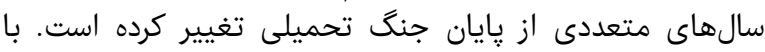

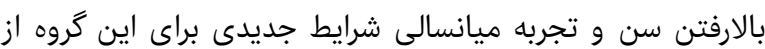

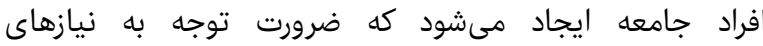

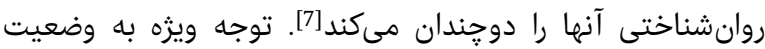

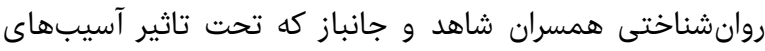

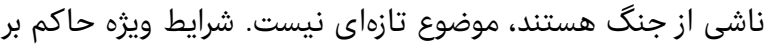

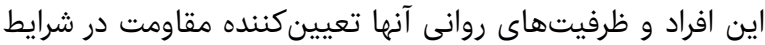

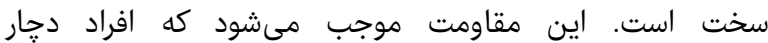

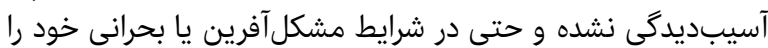

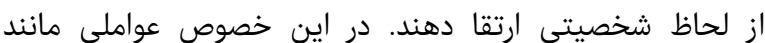

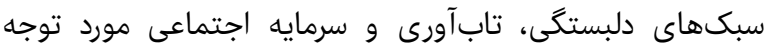
محققان بسيارى قرار گرفته است آست

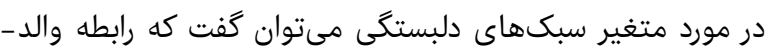

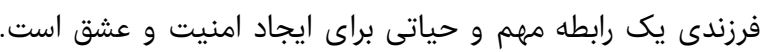

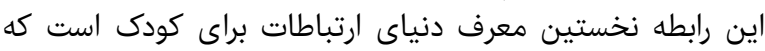

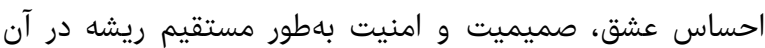

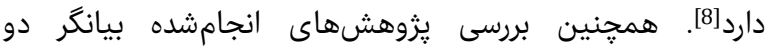

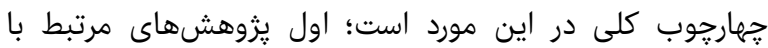

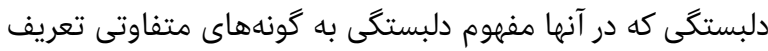

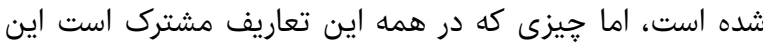

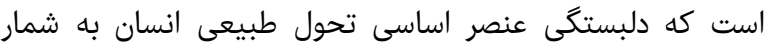

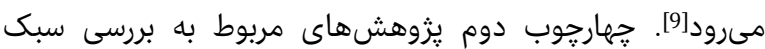

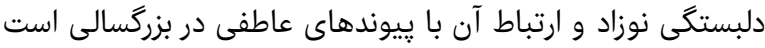

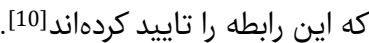

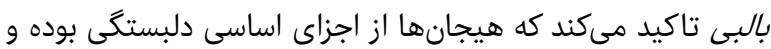

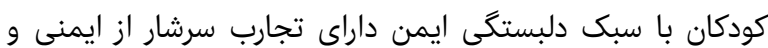

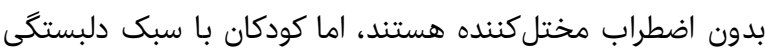

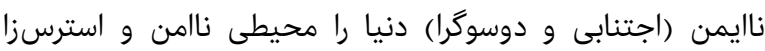

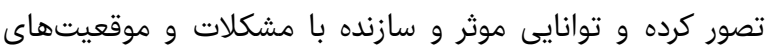

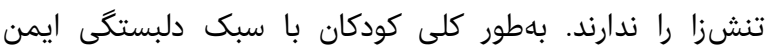

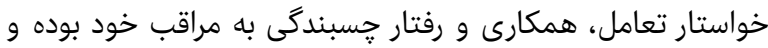

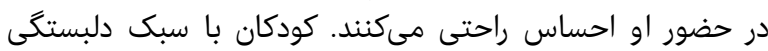

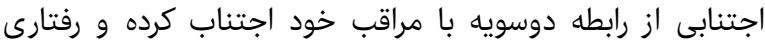

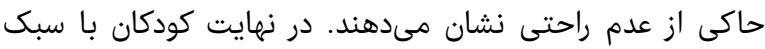

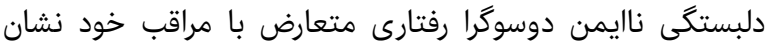

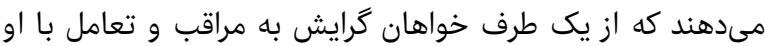

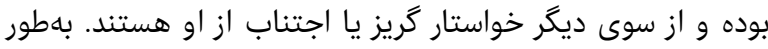

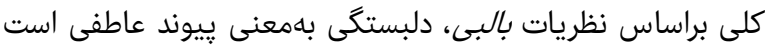

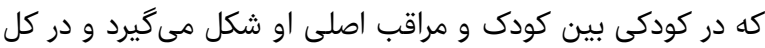

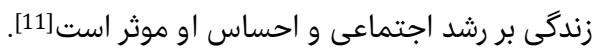

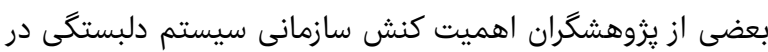

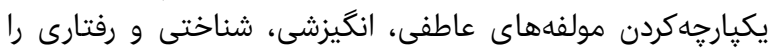

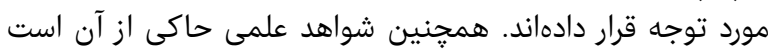

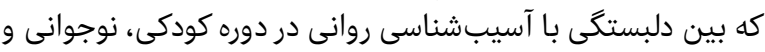

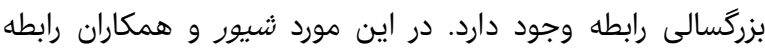

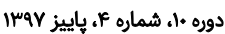

مقايسه سبكهاى دلبستگىى، تابآورى و و سرمايه

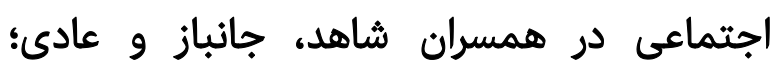
مطالعه موردى شهرستان اردبيل

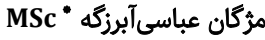

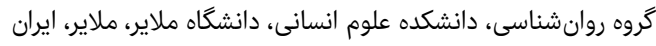

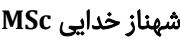

كروه روانشناسى، دانشكده علوم انسانى، واحد اردبيل، دانشگاه آزاد اسلامى،

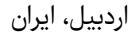

جكيده

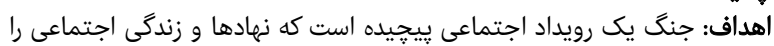

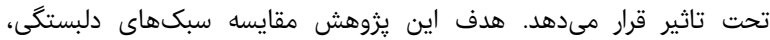

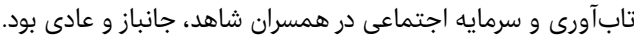

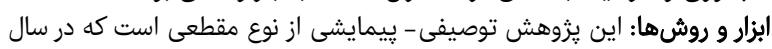

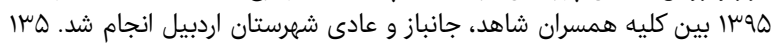

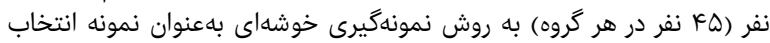

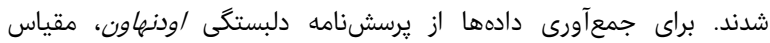

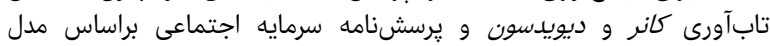

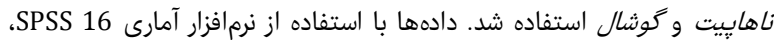

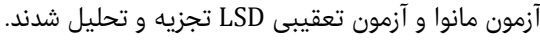

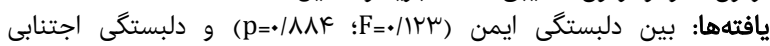

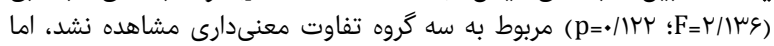

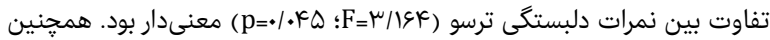

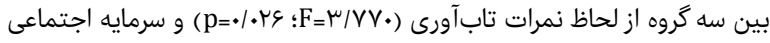
(p=/•/11 F=F/V|Q)

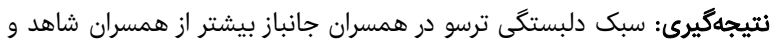

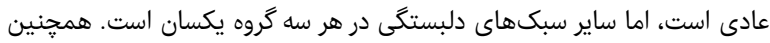

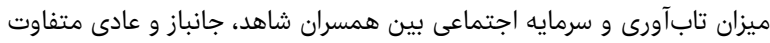

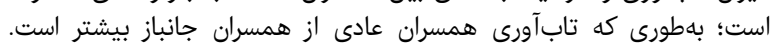

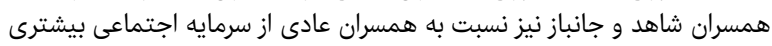

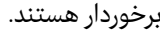
كليدواثهها: تابآورى، سرمايه اجتماعى، همسران، جانبازان

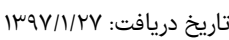

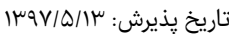

"نويسنده مسئول: mozhganabbasiabrazgah@gmail.com

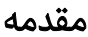

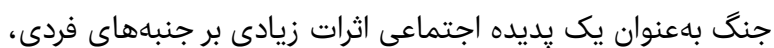

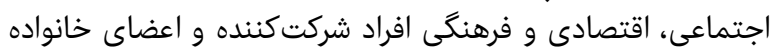

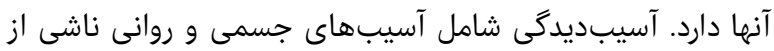

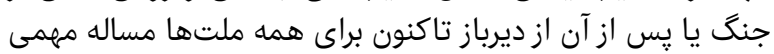

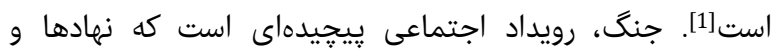

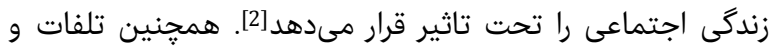

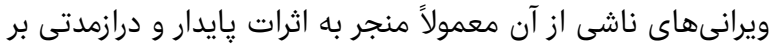

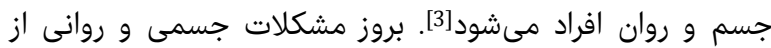

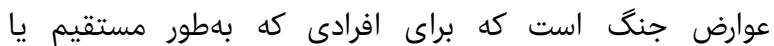

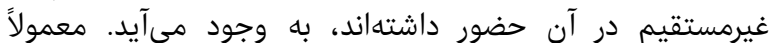

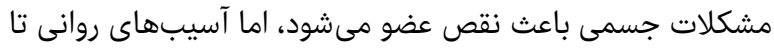

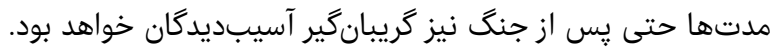

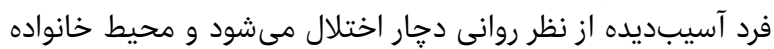

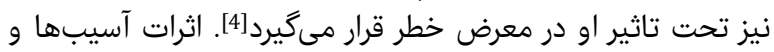

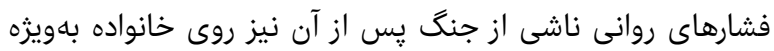

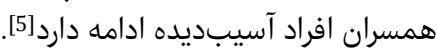
بسيارى از خانوادههاى شاهد و جانباز دركير بيامدهاى فاردان فاندان 


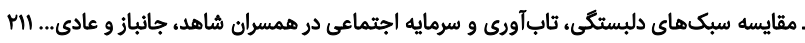

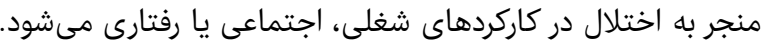

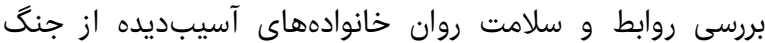

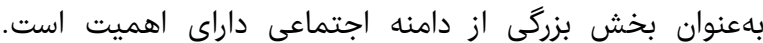

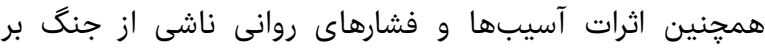

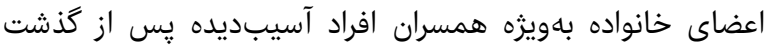

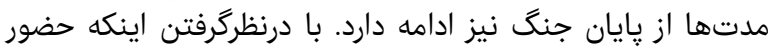

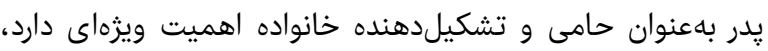

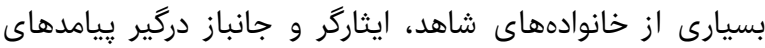

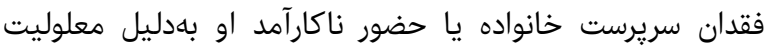

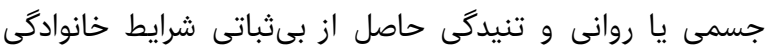

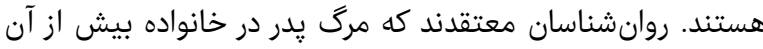

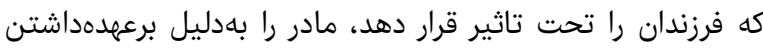

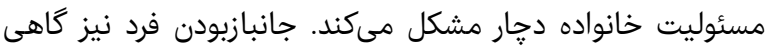

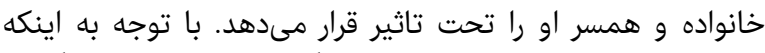

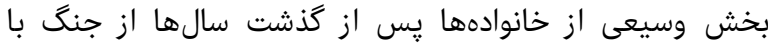

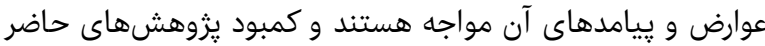

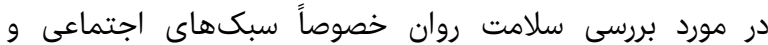

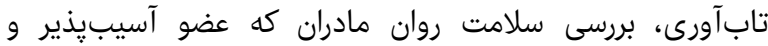

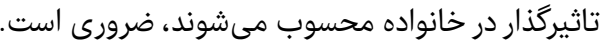

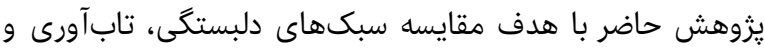

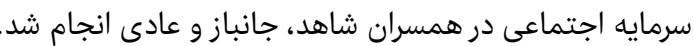

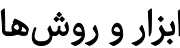

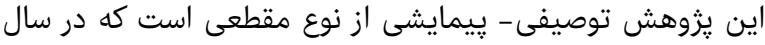

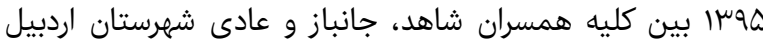

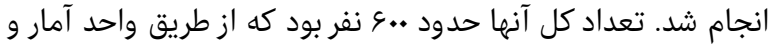

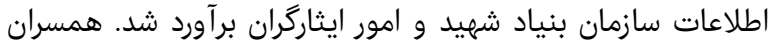

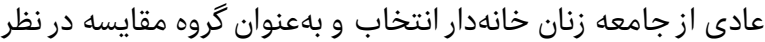

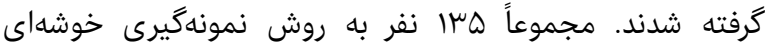

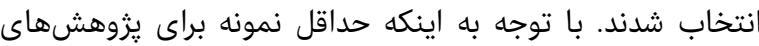

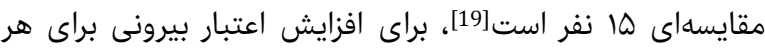

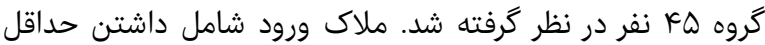

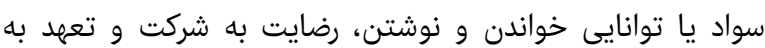

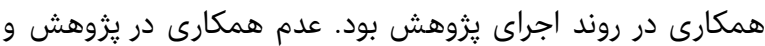

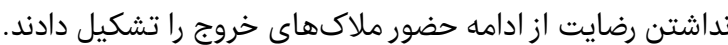

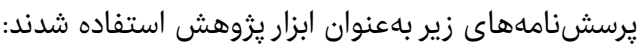

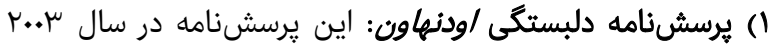

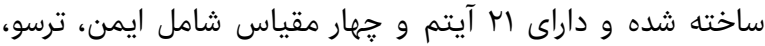

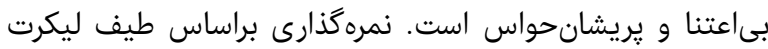

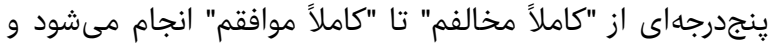

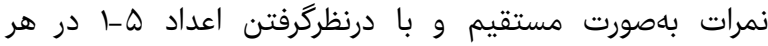

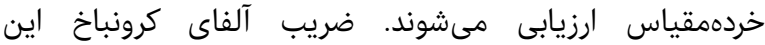

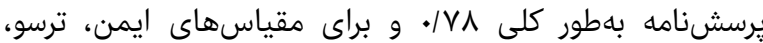

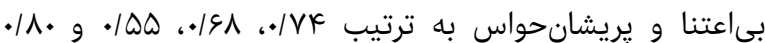

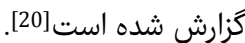

r) مقياس تابآورى كونور و ديويدسون (CD-RIS): اين مقياس

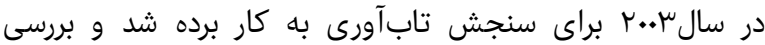

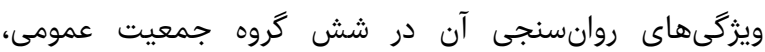

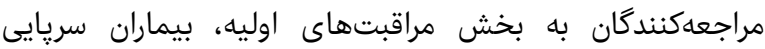

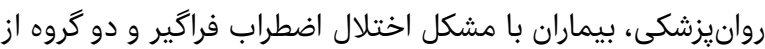

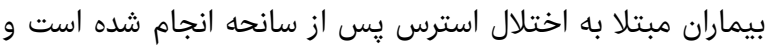

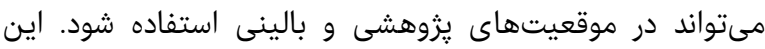

معنىارى بين سبكهاى دلبستگى با اختلالات خلقى، اضطرابى،

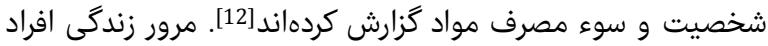

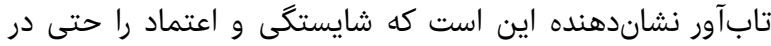

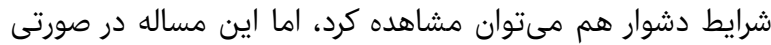

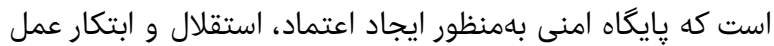

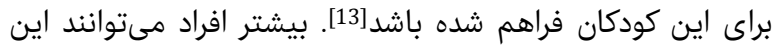

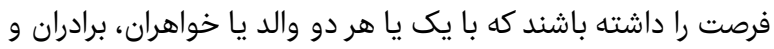

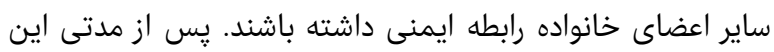

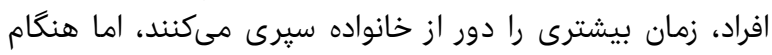

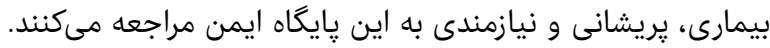

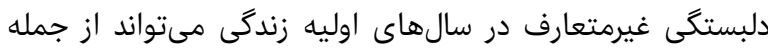

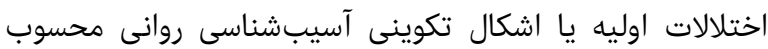
شود [14].

تابآورى نوعى حالت قابل توسعه در فرد است كه براساس آن

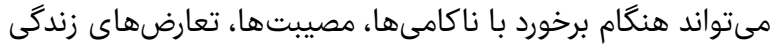

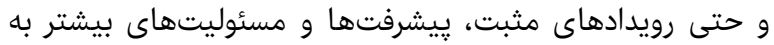

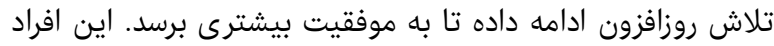

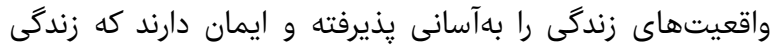

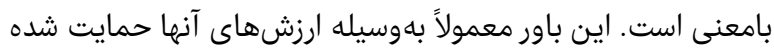

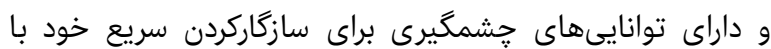
تغييرات بزرخ هستند [15].

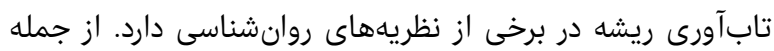

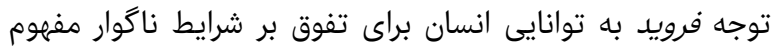

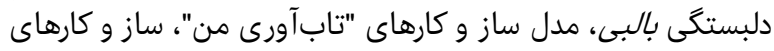

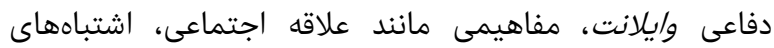

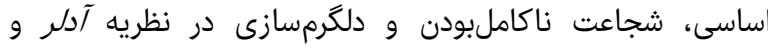

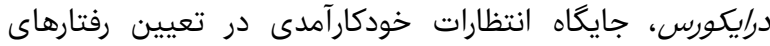

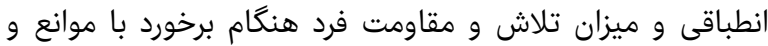

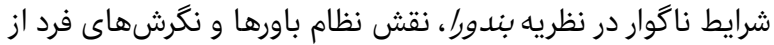

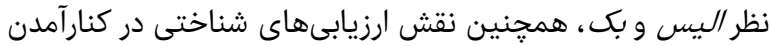

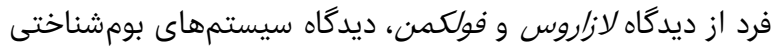

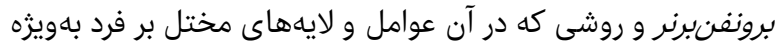

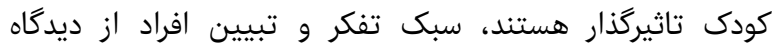

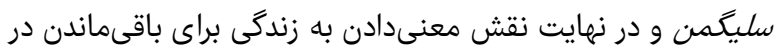

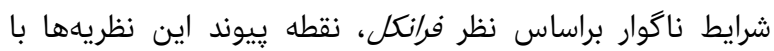

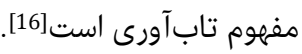

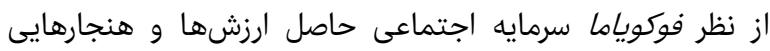

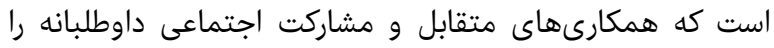

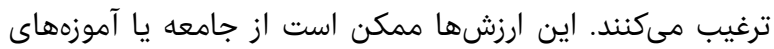

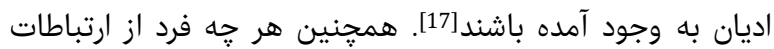

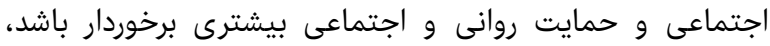

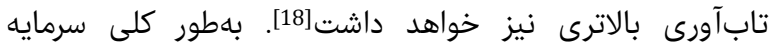

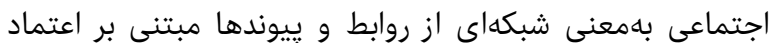

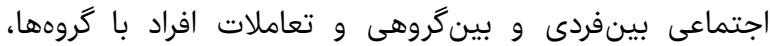

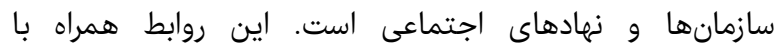

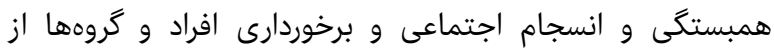

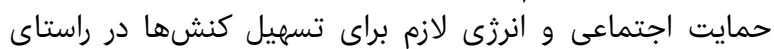

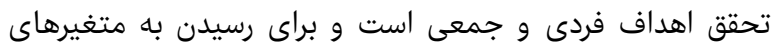

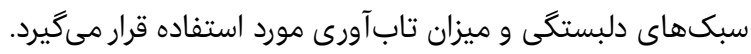

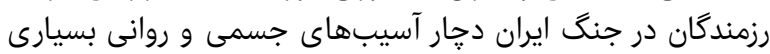

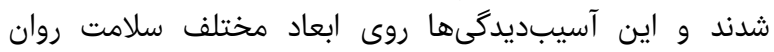

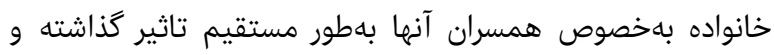


شاهد و جانباز از اين لحاظ تفاوت معنىدارى مشاهده نشد إدارد

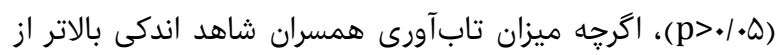

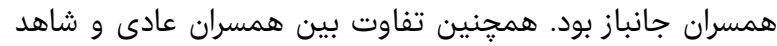

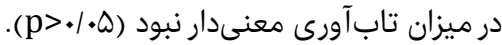

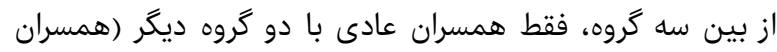

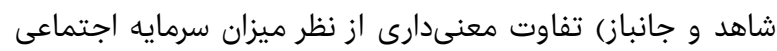

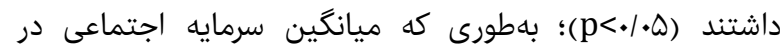

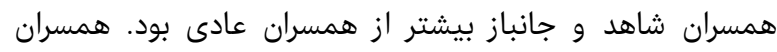

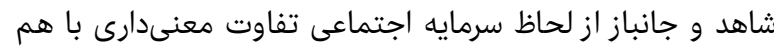

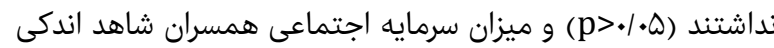

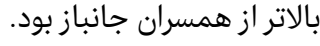

جدول () توزيع فراوانى مطلق و نسبى (اعداد داخل يرانتز درصد هستند)

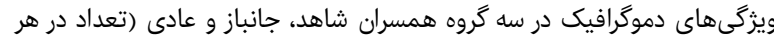

\begin{tabular}{|c|c|c|c|}
\hline همسران عادى & همسران جانباز & همسران شاهد & ويرگىها \\
\hline $\operatorname{Ir}(r T / V)$ & - & - & 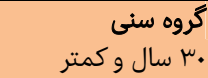 \\
\hline $19(\varepsilon r / r)$ & $T(1 \mu / \mu)$ & $r(\varepsilon / \varepsilon)$ & . ع-اس سال \\
\hline $1 \varepsilon(\mu \mid / I)$ & $\mu \varepsilon(V O / 7)$ & $r Y(7 . / \cdot)$ & 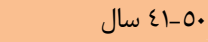 \\
\hline \multirow[t]{2}{*}{ - } & $0(11 / 1)$ & $17\left(\mathrm{HO}^{\circ} / 7\right)$ & بالاتر از •0 سال \\
\hline & & & وضعيت تحصيلى \\
\hline$r(\varepsilon / 0)$ & $V(10 / 0)$ & $19(\varepsilon r / r)$ & ابتدايى و نهضت \\
\hline - & $\operatorname{Ir}(r T / V)$ & $\varepsilon(1 / 9)$ & راهنمايى \\
\hline $1 \cdot(r \mu / r)$ & $9(Y \cdot / \cdot)$ & 1. (Yr/T) & دبيرستان و دييلم \\
\hline$r V(7 . / \bullet)$ & $I V(r V / \Lambda)$ & $V(10 / 7)$ & فوقدييلم و ليسانس \\
\hline \multirow[t]{2}{*}{$7(1 \mu / \mu)$} & - & $0(11 / 1)$ & فوقليسانس و بالاتر \\
\hline & & & وضعيت اشتغال \\
\hline $17(\mathrm{mo/7})$ & $\mu \mu(01 / 1)$ & $\mu_{\Lambda}(\Lambda \varepsilon / \varepsilon)$ & خانهدار \\
\hline $10(\mu \mu / \mu)$ & $V(10 / 7)$ & $\mu(T / V)$ & 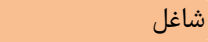 \\
\hline $1 \varepsilon(\mu / / I)$ & $10(\mu \mu / \mu)$ & $\varepsilon(1 / 9)$ & ساير \\
\hline
\end{tabular}

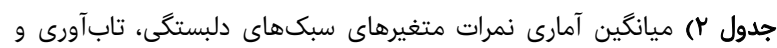

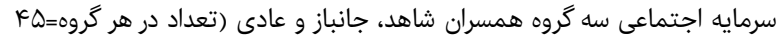

\begin{tabular}{|c|c|c|c|c|}
\hline جمع & همسران & همسران & هاهسران & 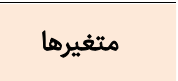 \\
\hline$\mu / \cdot q \pm \cdot / v$. & $\mu / \cdot 7 \pm \cdot / V\urcorner$ & $\mu / \vee \vee \pm \cdot / 7 \mu$ & $r / \| \mu_{ \pm} \cdot / V r$ & دلبستگى ايمن \\
\hline$r / 7 Y \pm \cdot / 7 /$ & $r / \varepsilon 0 \pm .100$ & $r / V 7 \pm . / 70$ & $r / 7 V \pm . / 7$. & دلبستگى ترسو \\
\hline$r / 0 \varepsilon \pm \cdot / T r$ & $r / \varepsilon r \pm \cdot / 0 V$ & $r / 79 \pm . / 77$ & r/Ort.109 & دلبستكى اجتنابى \\
\hline$r / 91 \pm \cdot / 0 r$ & $\varepsilon / \cdot V \pm \cdot 10 r$ & r/VA \pm . $/ 0 \mathrm{r}$ & భ/८ $\pm \pm / \varepsilon q$ & تابآورى \\
\hline$. / 7 V \pm . / 11$ & $. / 7 \varepsilon \pm \cdot / 11$ & $\cdot / 7 \wedge \pm \cdot / 11$ & $\cdot / V \cdot \pm \cdot / I I$ & سرمايه اجتماعى \\
\hline
\end{tabular}

بين همسران عادى و حانباز از لحاظ سبك دلبستخى ترسو تفاوت

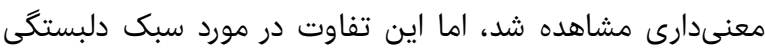

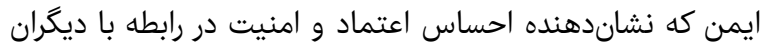

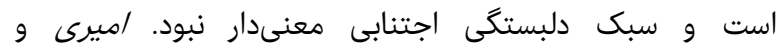

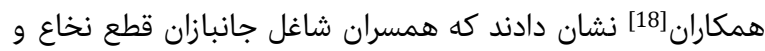

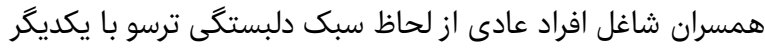

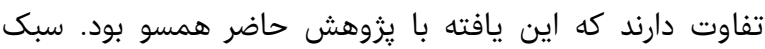

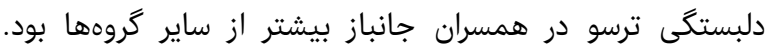

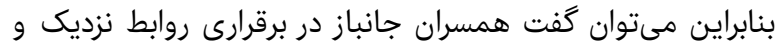

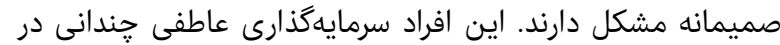

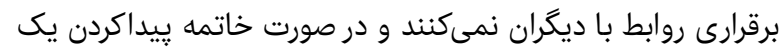

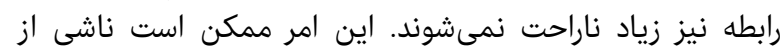

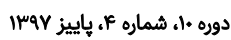

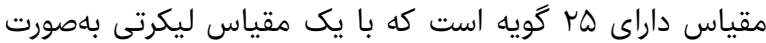

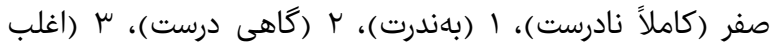

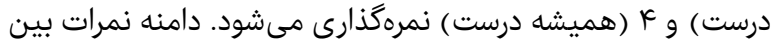

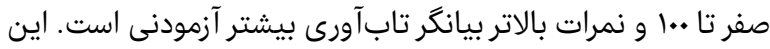

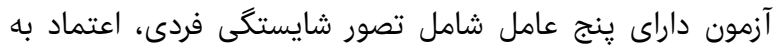

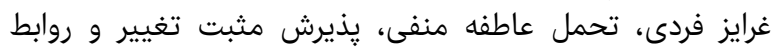

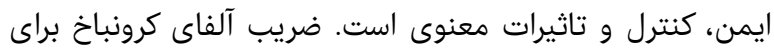

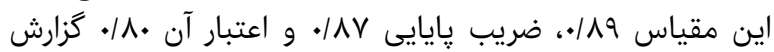

شده است[21]. - است.

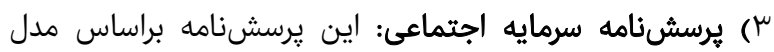

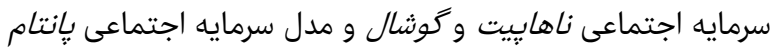

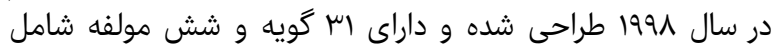

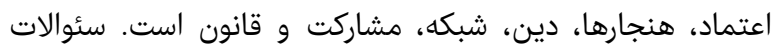

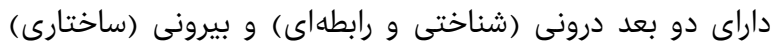

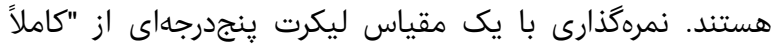

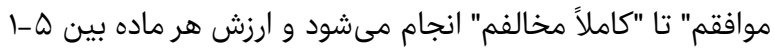

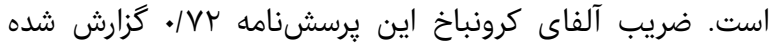

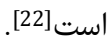

براى جمعآورى اطلاعات ليست اسامى و آدرس همسران شاهد از

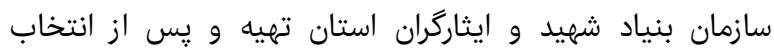

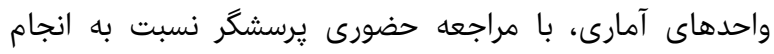

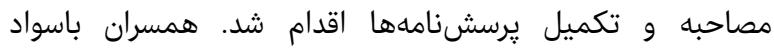

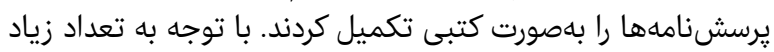

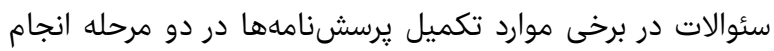

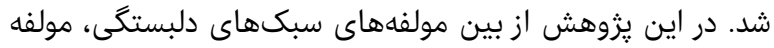

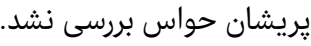

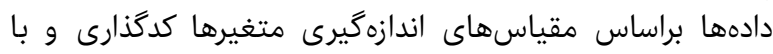

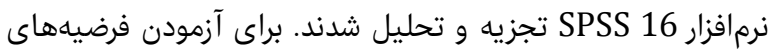

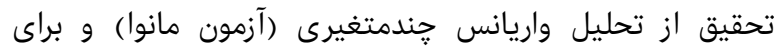

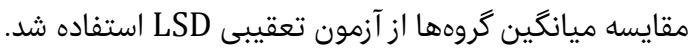

يافتهها

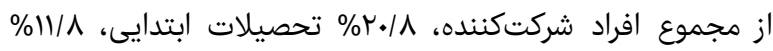

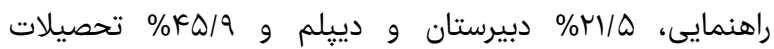

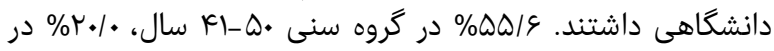

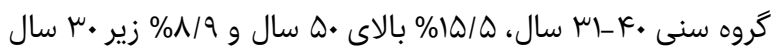

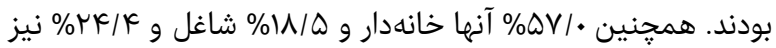

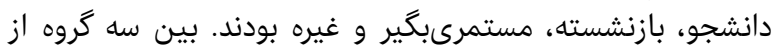

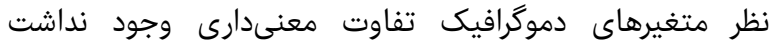

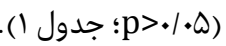

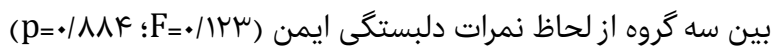

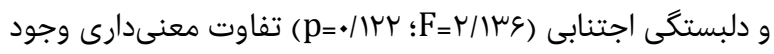

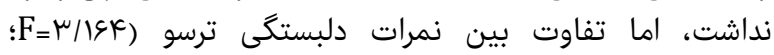

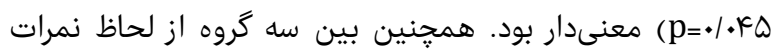

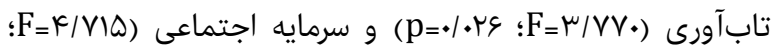

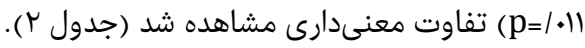

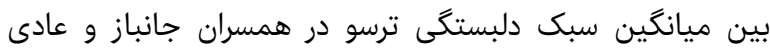

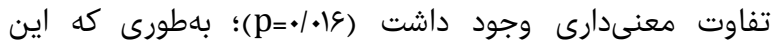

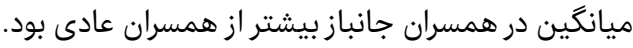

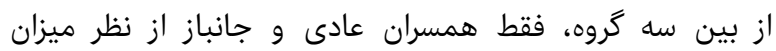

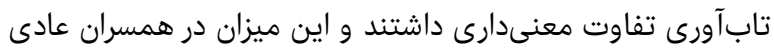

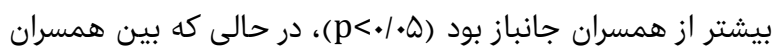




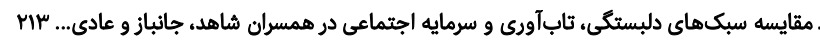

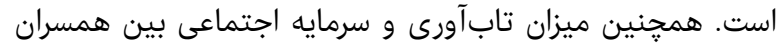

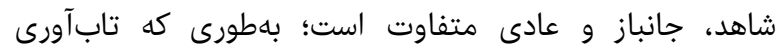

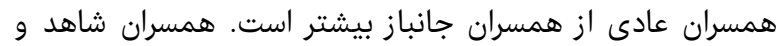

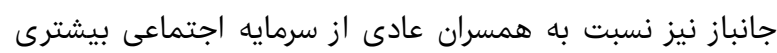
برخوردار هستند.

تشكر و قدردانى: از كليه همسران شاهد و جانباز شركت كننده در

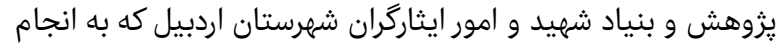

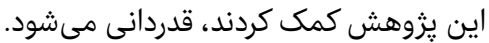

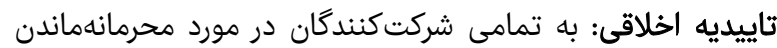

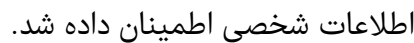

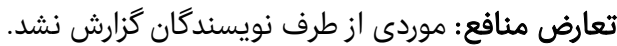

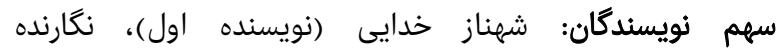

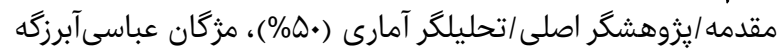

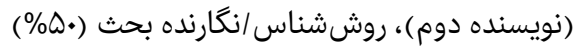

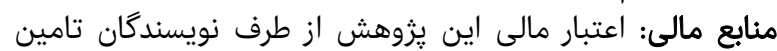

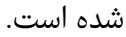

منابع

1- Masoumi M, Soroush MR, Modirian E. Evaluation of psychiatric problems among war-related bilateral upper limb amputees. J Mil Med. 2008;10(3):185-92. [Persian] 2- Rostamalizadeh V, Ghasemiardahaee A. Impacts and implications of social-demographic immigration of imposed war in Iranian society. Holy Def Res Cent. 2012;1(2):59-79. [Persian]

3- Behdani F, Fayyazi Bordbar MR, Hebrani P, Talebi M, Kohestani L. Psychiatric symptoms in chemical and somatic combat veterans. J Mil Med. 2009;11(1):31-5. [Persian]

4- Nabatian E, Ghamarani A, Zakerian M, Mahdizadeh I. Relationship between spiritual health with quality of life veterans and disabled Birjand. Iran J War Public Health. 2013;5(2):35-9. [Persian]

5- Mardani Hamouleh M, Borimnejhad L, Seyyed Fatemi $\mathrm{N}$, Taghavi T. Correlation between general health and quality of life in caregivers of veterans with spinal cord injury. Iran J War Public Health. 2014;6(3):81-6. [Persian]

6- Akbari Z, Vafaei T, Khosravi S. Relationship between attachment styles and the resiliency amount of veterans wives. Iran J War Public Health. 2011;3(2):12-7. [Persian]

7- Safavi M, Mahmoudi M, Akbarnatajbisheh K. Assessment of relationship quality of life and coping skills in spouses of chemical devotees with pulmonary complications due to sulfur mustard in Tehran in 2006. Daneshvar Med. 2010;17(87):9-18. [Persian]

8- Carnes Holt K. Child-parent relationship therapy for adoptive families. Fam J. 2012;20(4):419-26.

9- Malekpour M. Effect of attachment on early and later development. Br J Dev Disabil. 2007;53(105):81-95.

10- Hazan C, Shaver P. Romantic love conceptualized as an attachment process. J Personal Soc Psychol. 1987;52(3):511-24.

11- Van Den Dries L, Juffer F, Van Ijzendoorn $\mathrm{MH}$, Bakermans-Kranenburg MJ. Fostering security? A metaanalysis of attachment in adopted children. Child Youth Serv Rev. 2009;31(3):410-21.

12- Besharat MA, Irvani M, Sharifi M. An investigating the relationship between attachment styles and defense mechanisms. J Psychol. 2001;5(3-19):277-89. [Persian]
مشكلاتى باشد كه همسران جانبازان در رسيدگى به فردان جانباز و و

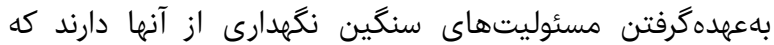

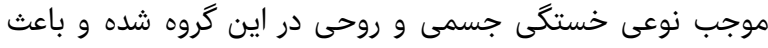
مى شود كه فرصت و حوصله برقرارى روابط با افراد بيشترى نداشته باشند. بين سه كروه از نظر ميزان تابآورى تفاوت معنى دابدارى وجود

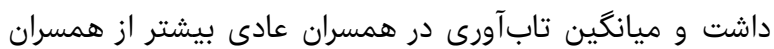

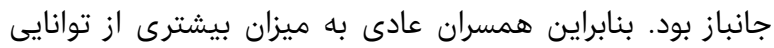

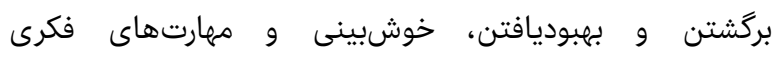

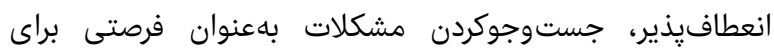

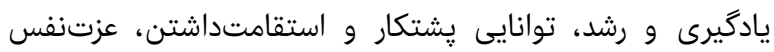

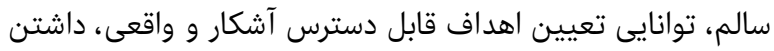

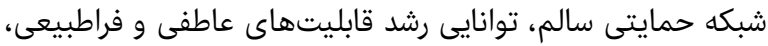

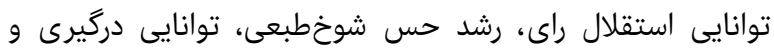

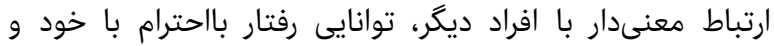

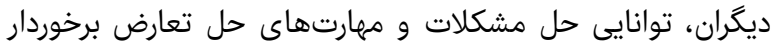

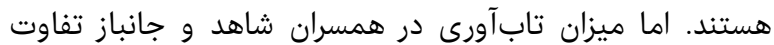

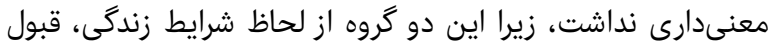

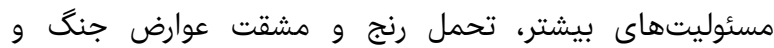

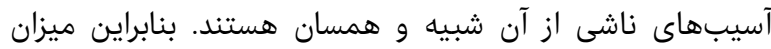

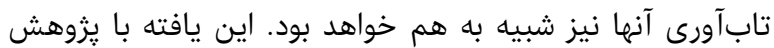

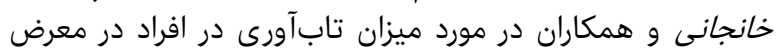

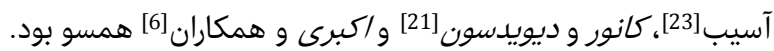

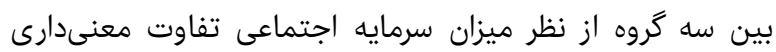

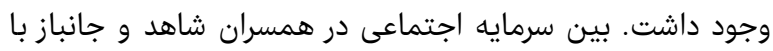

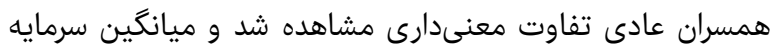

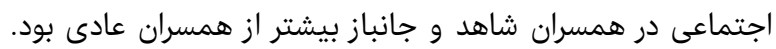

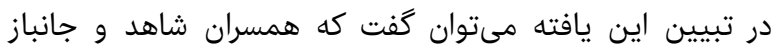

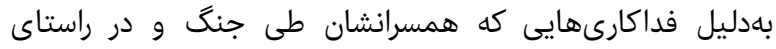

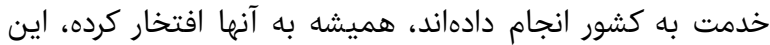

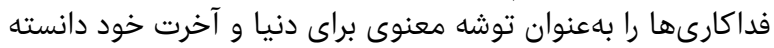

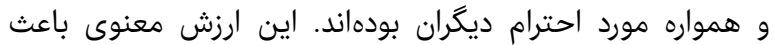

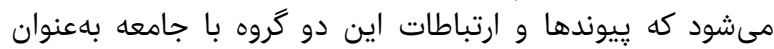

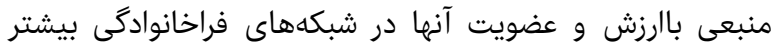

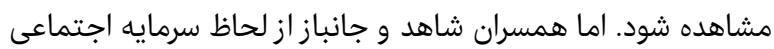

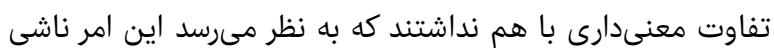

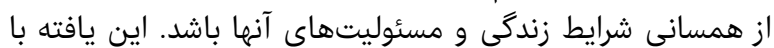

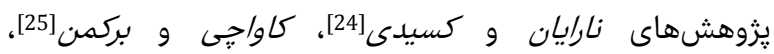

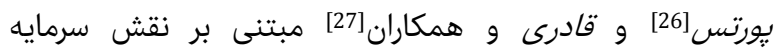

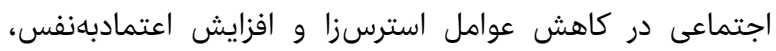

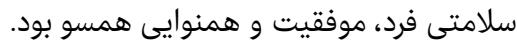

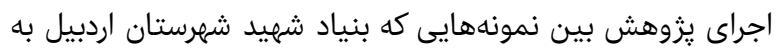

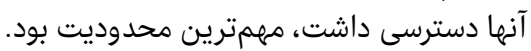

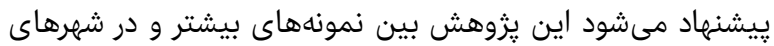

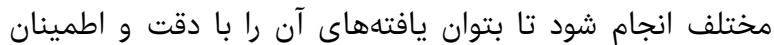

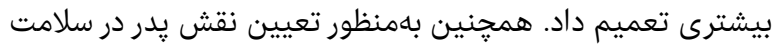

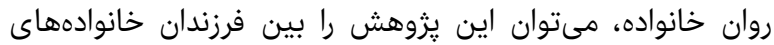
شهدا و جانبازان نيز انجام داده، مئوان إن

\section{نتيجه ديرى}

سبك دلبستخى ترسو در همسران جانباز بيشتر از همسران شاهد و و دئي عادى است، اما ساير سبكهاى دلبستخى در در هر سه گروه يكسان 
Ardabil: Mohaghegh Ardabili Publication. 2010; p. 159. [Persian]

21- Conner KM, Davidson JRT. Development of a new resilience scale: The Connor-Davidson resilience scale (CD-RISC). Depress Anxiety. 2003;18(2):76-82.

22- Putnam R, Coleman J, Este K, Bourdieu P, Fukuyama F, Porz A, et al. Social Capital: Trust, democracy and development. $1^{\text {st }}$ Edition. Khakbaz A, Pooyan $\mathrm{H}$, translators. Tehran: Shirazeh research publication; 2005. [Persian]

23- Khanjani M, Sohrabi F, Aazami Y. The effectiveness of resilience and stress management training program on psychological well-being, meaning of life, optimism, and satisfaction of life in female-headed households. Iran J Psychiatr Nurs. 2018;6(2):1-11. [Persian]

24- Narayan D, Cassidy MF. A dimensional approach to measuring social capital: Development and validation of a social capital inventory. Curr Sociol. 2001;49(2):59102.

25- Kawachi I, Berkman LF. Social ties and mental health. J Urban health. 2001;78(3):458-67.

26- Portes A. Social capital: Its origins and applications in modern sociology. Annu Rev Sociol. 1998;24(1):1-24.

27- Ghaderi M, Maleki A, Haghjo M. The role of social capital in promoting a healthy lifestyle among people with coronary artery disease. Iran J Cardiovasc Nurs. 2015;4(32):24-35. [Persian] Smith RS. Overcoming the odds: High risk children from birth to adulthood. Ithaca and London: Cornell University Press; 1992. p. 280 14- Gobari Bonab B, Steyri Z. Comparison of personality traits and attachment style in mothers of children with disturbed self-deprivation and mothers of normal children. Iran J Except Child. 2006;6(3):787-804. [Persian]

15- Samani S, Jokar B, Sahragard N. Effects of resilience on mental health and life satisfaction. Iran J Psychiatry Clin Psychol. 2007;13(3):290-5. [Persian]

16- Kaveh M, Alizadeh H, Delavar A, Barajali A. Development of a resilience fostering program against stress and its impact on quality of life components in parents of children with mild intellectual disability. J Except Child. 2011;11(2):119-40. [Persian]

17- Rouhani $\mathrm{H}$. The capital of the family, the foundation stone of social capital. Rahbord. 2011;20(59):7-46. [Persian]

18- Amiri M, Bordbar S, Kateb Z. Comparison of the mental status of working wives of disabled veterans with their spouse working in normal people. J Psychol. 2005;9(1):22-33. [Persian]

19- Delavar A. Research methods in psychology and educational sciences. Tehran: Virayesh; 2005. p. 298. [Persian]

20- Narimani M, Ahadi B. Personality tests. $1^{\text {st }}$ Edition. 